\title{
Engineering Value in Healthcare: Applying Failure Modes and Effects Analysis to Value Based Purchasing
}

\author{
Amy Cress ${ }^{1}$, Lee Revere ${ }^{2 *}$, Osama Mikhail ${ }^{3}$, Lisa Pompeii ${ }^{3}$ and Debora Simmons ${ }^{4}$ \\ ${ }^{1}$ Texas Children's Hospital, The Woodlands, TX \\ ${ }^{2}$ University of Texas Health Science Center at Houston, School of Public Health, Houston, TX \\ ${ }^{3}$ University of Texas Health Science Center at Houston, Houston, TX \\ ${ }^{4}$ University of Texas Health Science Center at Houston, School of Biomedical Sciences Houston, TX
}

\begin{abstract}
The Centers for Medicare and Medicaid Services (CMS) introduced the hospital Value Based Purchasing (VBP) program in 2012, providing a definition and structure for measuring value in hospital-based healthcare services. Under this new program, CMS withholds a percentage of reimbursement from each participating hospital. CMS then distributes withholds back to hospitals based on VBP total performance scores (TPS), which are weighted calculations that include scores on multiple measures such as clinical process of care and patient satisfaction. Currently, hospitals have no easily applied method for prioritizing which measures of VBP to improve in order to maximize the hospital's reimbursement of withhold. This research develops and pilots an objective method, Value Failure Modes and Effects Analysis (VFMEA), for hospitals to target VBP measures for improvement by analyzing current performance and optimizing limited resources. VFMEA provides hospital administrators needed insight to reliably prioritize VBP measures for improvement and to direct resources toward improvement that will produce the largest gain in the hospital's TPS, and thus reimbursement of withhold.
\end{abstract}

Keywords: Value based purchasing; Value; CMS

Abbreviations: CMS: The Centers for Medicare and Medicaid Services; FMEA: Failure Modes and Effects Analysis; HCAHPS: Hospital Consumer Assessment of Healthcare Providers and Systems; HFMEA: Healthcare Failure Modes and Effects Analysis; SCIP: The Surgical Care Improvement Project; TPS: Total Performance Scores; VA: Veterans Affairs; VBP: Value Based Purchasing; VFMEA: Value Failure Modes and Effects Analysis; VIS: Value Impact Score

\section{Introduction}

Many healthcare leaders have often said "No Margin; No Mission"; however, in today's age of increasing quality of care regulation, the new mantra has become "No outcome; No income." The new mantra is due to the recent Center for Medicare and Medicaid Services (CMS) introduction of the Value Based Purchasing (VBP) Program, a program designed to tie hospital income to quality outcomes. VBP provides hospitals with financial incentives for improving performance on defined measures of quality and patient satisfaction. CMS withholds money from hospital reimbursements and rewards hospitals through incentive payments for performance at or above the performance standard [1]. VBP does not provide guidance on how a hospital should achieve these outcomes. Instead, local facilities have the flexibility to identify, improve and implement process changes they deem necessary to impact their performance scores and optimize their incentive payments [2]. This task is daunting for many hospital leaders because there is no systematic and objective method for healthcare organizations to compare performance and prioritize improvements on measures across multiple domains.

Methods adapted from outside healthcare, such as the Failure Modes and Effects Analysis (FMEA) used in engineering and manufacturing, may provide healthcare leaders a means to compare performance measures and prioritize opportunities for improvements that will directly impact their VBP outcomes and resulting payments. This research creates a modified FMEA methodology that enables a hospital to assess the multiple performance domains measured in VBP and prioritize improvement opportunities to maximize CMS reimbursement. The new methodology, referred to as Value Failure Modes and Effects Analysis (VFMEA), provides a proactive method for hospital leaders to identify the measures specific to a facility that will maximize the incentive payment received.

\section{Literature Review}

The VBP program was officially launched in fiscal year 2013 and was applied to patients discharged after October 1, 2012 (the first day of fiscal year 2013) [3]. In the VBP program, CMS withholds a percentage of monetary reimbursement from each of the participating hospitals [3]. In fiscal year 2014, the withhold was $1.25 \%$ and will increase by $0.25 \%$ each year through fiscal year 2017 [3]. Withholds are distributed to hospitals based on individual hospital performance across multiple measures, which are aggregated results of patient outcomes, organized into weighted domains [3]. Each domain score is weighted to calculate a total performance score (TPS) [4]. Based on the TPS, CMS either withholds money from hospital reimbursements or provides an incentive payment for performance at or above the established TPS standard [1]. The program changes each fiscal year by changing the domains and measures included, changing domain weights, increasing withholds, and modifying incentive payments based on performance [3]. Table 1 lists the domains and measures for fiscal year 2014, the study period [3]; Table 2 shows VBP domains, measures, and weighting by fiscal year.

\section{Value Based Purchasing}

A fundamental understanding of the architecture of VBP domains and measures provides the basis for understanding how CMS scores a hospital's performance. A hospital's performance in the VBP

${ }^{*}$ Corresponding author: Lee Revere, SUniversity of Texas Health Science Center at Houston, School of Public Health, Houston, TX, Tel: + 281-701-0030; E-mail: frances.lee.revere@uth.tmc.edu

Received December 19, 2016; Accepted January 20, 2017; Published January 27, 2017

Citation: Cress A, Revere L, Mikhail O, Pompeii L, Simmons D (2017) Engineering Value in Healthcare: Applying Failure Modes and Effects Analysis to Value Based Purchasing. Health Econ Outcome Res Open Access 3: 124. doi: 10.4172/2471268x/1000124

Copyright: ( 2017 Revere F, et al. This is an open-access article distributed under the terms of the Creative Commons Attribution License, which permits unrestricted use, distribution, and reproduction in any medium, provided the original author and source are credited. 
Citation: Cress A, Revere L, Mikhail O, Pompeii L, Simmons D (2017) Engineering Value in Healthcare: Applying Failure Modes and Effects Analysis to Value Based Purchasing. Health Econ Outcome Res Open Access 3: 124. doi: 10.4172/2471-268x/1000124

Page 2 of 6

\begin{tabular}{|c|c|c|c|}
\hline Fiscal Year $^{1}$ & Domain $^{2}$ & Measure ID ${ }^{3}$ & Measure (M) ${ }^{4}$ \\
\hline \multirow{13}{*}{ 2013-2015 } & \multirow{13}{*}{ Clinical Processes of Care } & AMI-7a & Fibrinolytic therapy received within 30 min of hospital arrival \\
\hline & & AMI-8a & Primary $\mathrm{PCl}$ received within 90 min of hospital arrival \\
\hline & & $\mathrm{HF}-1$ & Discharge instructions \\
\hline & & PN-3b & $\begin{array}{l}\text { Blood cultures performed in the Emergency Department prior to initial } \\
\text { antibiotic received in hospital }\end{array}$ \\
\hline & & PN-6 & $\begin{array}{l}\text { Initial antibiotic selection for community-acquired pneumonia in } \\
\text { immunocompetent patients }\end{array}$ \\
\hline & & SCIP-Inf-1 & Prophylactic antibiotic received within one hour prior to surgical incision \\
\hline & & SCIP-Inf-2 & Prophylactic antibiotic selection for surgical patients \\
\hline & & SCIP-Inf-3 & Prophylactic antibiotics discontinued within $24 \mathrm{~h}$ after surgery end time \\
\hline & & SCIP-Inf-4 & $\begin{array}{l}\text { Cardiac surgery patients with controlled 6:00 a.m. postoperative serum } \\
\text { glucose }\end{array}$ \\
\hline & & $\begin{array}{l}\text { SCIP-Inf-9 } \\
\text { (FY 2014-2015 only) }\end{array}$ & Urinary catheter removal on postoperative day 1 or postoperative day 2 \\
\hline & & SCIP-Card-2 & $\begin{array}{l}\text { Surgery patients on a beta-blocker prior to arrival who received a beta- } \\
\text { blocker during the perioperative period }\end{array}$ \\
\hline & & SCIP-VTE-1 (for FY 2013-2014 only) & $\begin{array}{l}\text { Surgery patients with recommended venous thromboembolism } \\
\text { prophylaxis ordered }\end{array}$ \\
\hline & & SCIP-VTE-2 & $\begin{array}{l}\text { Surgery patients who received appropriate venous thromboembolism } \\
\text { prophylaxis within } 24 \mathrm{~h} \text { prior to surgery to } 24 \mathrm{~h} \text { after surgery }\end{array}$ \\
\hline \multirow{8}{*}{ 2013-2015 } & \multirow{8}{*}{$\begin{array}{l}\text { Patient Experience of Care } \\
\text { (HCAHPS) }\end{array}$} & \multirow{8}{*}{ Measure ID not used for HCAHPS } & Nurse communication \\
\hline & & & Doctor communication \\
\hline & & & Hospital staff responsiveness \\
\hline & & & Pain management \\
\hline & & & Medicine communication \\
\hline & & & Hospital cleanliness and quietness \\
\hline & & & Discharge information \\
\hline & & & Overall hospital rating \\
\hline \multirow{5}{*}{ 2014-2105 } & \multirow{5}{*}{ Outcomes } & MORT-30-AMI & Acute myocardial infarction 30-day mortality rate \\
\hline & & MORT-30-HF & Heart failure 30 -day mortality rate \\
\hline & & MORT-30-PN & Pneumonia 30-day mortality rate \\
\hline & & $\begin{array}{l}\text { AHRQ PSI-90 } \\
\text { (FY15 Only) }\end{array}$ & Complication/patient safety for selected indicators \\
\hline & & $\begin{array}{l}\text { CLABSI } \\
\text { (FY15 Only) }\end{array}$ & Central line-associated blood stream infection \\
\hline 2015 & Efficiency & MSBP-1 & Medicare spending per beneficiary \\
\hline
\end{tabular}

Table 1: Value based purchasing domains and measures by fiscal year.

\begin{tabular}{|c|c|c|c|}
\hline Fiscal Year & Withhold & Measures included in Value Based Purchasing & Domain Weigh \\
\hline \multirow{2}{*}{2013} & \multirow{2}{*}{$1.0 \%$} & Clinical Processes of Care & $70 \%$ \\
\hline & & Patient Experience of Care (HCAHPS) & $30 \%$ \\
\hline \multirow{3}{*}{2014} & \multirow{3}{*}{$1.25 \%$} & Clinical Processes of Care & $45 \%$ \\
\hline & & Patient Experience of Care (HCAHPS) & $30 \%$ \\
\hline & & Outcomes & $25 \%$ \\
\hline \multirow{4}{*}{2015} & \multirow{4}{*}{$1.5 \%$} & Clinical Processes of Care & $20 \%$ \\
\hline & & Patient Experience of Care (HCAHPS) & $30 \%$ \\
\hline & & Outcomes & $30 \%$ \\
\hline & & Efficiency & $20 \%$ \\
\hline
\end{tabular}

Table 2: Value based purchasing domains by fiscal year.

program is reported as the TPS [3], calculated from both the hospital's performance achievement relative to a CMS benchmark and the hospital's performance improvement relative to the hospital's previous performance [3]. There is no minimum TPS and the scores vary based on the measures that are included in the fiscal year and those that are applicable to the hospital [3]. Measures included in a particular fiscal year are summed to weighted domain totals and are used to calculate the hospital's TPS. Changes in domain weights by fiscal year are shown in 
Table 2. For each measure, an achievement score and an improvement score are calculated and the larger of the two is used in the domain score [4]. The measure scores are publicly reported as $n$ points earned out of 10 possible points for achievement and $n$ points earned out of 9 possible points for improvement [4].

Incentive payments are based on a hospital's individual performance during the performance period, which is a specific period of time when data are collected [4]. CMS compares the TPS of participating hospitals and generates a linear function to determine the incentive payment for each hospital participating in the VBP Program. Hospitals with performance at or above the performance standard (benchmark) receive an incentive payment and hospitals that are below the benchmark do not receive an incentive payment [1]. The performance standard changes for each cycle of VBP, making it difficult for healthcare leaders to predict the TPS needed to receive an incentive payment.

\section{Healthcare Failure Mode Effect Analysis}

The U.S. Department of Veterans Affairs Administration (VA) first adapted FMEA for identifying risk of failure in single areas of healthcare in a tool called the Healthcare Failure Modes and Effects Analysis (HFMEA) [5, 6]. The VA describes HFMEA as a systematic, engineering-based, and proactive method for identifying "potential failure modes" within a process or system [6]. The VA methodology and tools are used widely within the healthcare industry and are recognized by The Joint Commission, an accrediting body for healthcare organizations, as a robust tool for proactively identifying patient safety risks [7]. The VA National Center for Patient Safety uses the adapted HFMEA in all 153 VA hospitals [6].

In an HFMEA, systems are divided into sub processes and potential failure modes are identified for each sub process [8]. For example, a failure mode might be incorrect medication dose or incomplete documentation. If any sub process fails, the whole system is vulnerable to failure. The same overall approach can be used to evaluate VBP as a system; that is, to identify a hospital's weakness via understanding potential weaknesses in individual VBP measures. In VBP, domains (HCHAPS, clinical processes of care, and outcomes) are equivalent to sub processes and measures (SCIP, communication with nurses, etc. shown in Table 1) are equivalent to the failure modes. Therefore, if a hospital fails on one of the measures, the domain is weakened and is vulnerable to failure. Addressing the most vulnerable aspects provides the most reinforcement to the system and the greatest potential improvement in value. Figure 1 depicts how the HFMEA model is translated to VBP. In this example, clinical process of care is vulnerable to failure if the hospital does not perform well on the individual measures of heart failure discharge instruction or does not give prophylactic antibiotics within 60 minutes before surgical incision.

\section{Statement of the Problem}

Currently, there is no objective method for hospital leaders to prioritize the VBP measures and identify those opportunities that can have the largest impact on their TPS. It is difficult for leaders to prioritize VBP measures because of multiple domains, multiple measures, changes implemented every fiscal year, changing break-even points every fiscal year, and the challenges of calculating the TPS. Hospitals need a reliable method to identify opportunities and prioritize quality improvements to improve individual VBP measures [9]. Looking outside of the healthcare industry at the FMEA methodology provides opportunity for identifying a systematic, structured, and objective method for prioritizing improvement opportunities based on their impact on VBP. Using the CMS standard data definitions for VBP makes the VFMEA methodology developed in this research broadly applicable for any hospital because it can be easily applied, does not require any financial investment to implement and can be tailored to individual facilities

\section{Methods}

This research develops the VFMEA process and demonstrates its applicability to identify the VBP measures most needing improvement. The VFMEA is adapted from the HFMEA process developed by the VA. In HFMEA the basic steps are:

1. Define the topic.

2. Assemble the team.

3. Graphically describe the process.

4. Conduct a hazard analysis.

5. Identify actions and outcomes measures [7].

The VFMEA methodology prioritizes the measures of the CMS VBP structure to prioritize performance improvement opportunities among the measures of VBP so that a hospital can maximize its TPS. This research focused on domains and associated measures for fiscal year 2014, including clinical process of care, HCAHPS results, and outcomes. Through application of these five basic steps to VBP, the VFMEA can be reduced to conducting the hazard analysis and prioritizing the VBP measures. The hazard analysis is broken into two key steps: 1) calculating the Value Impact Score for each element and 2) completing the decision tree and action types. The basic steps for the VFMEA are outlined in Figure 2.

\section{Step 1: Calculate VFMEA value impact score for each element}

In the VA's HFMEA, the hazard score is a multiplier of the severity of the failure and the likelihood that the failure will occur. To apply VA HFMEA methodology to VBP, it was necessary to modify the hazard score. The modified hazard score is called the Value Impact Score (VIS). The VIS is a multiplier of the gap between the hospital's performance and benchmark and the VBP weight, as shown in Figure 3.

CMS reports performance for each measure on a publicly available, comparative website as an achievement score out of 10 points and an improvement score out of 9 points. The gap was recorded as the larger of the difference between the maximum 10 points available for achievement or the maximum 9 points available for improvement. For example, if a hospital earned 8 points on the achievement score (a 2-point gap) and 6 points on the improvement score (a 3-point gap), the larger difference ( 3 points) from the improvement score would be recorded. Calculating the gap as the larger of the two differences makes the method more broadly applicable to hospitals, regardless of current performance. It also makes the methodology robust to changes in benchmarks over multiple fiscal years. Furthermore, using data provided by CMS reduces the burden on the hospital to know or understand all of the intricate details of VBP.

The VBP weight is also needed to calculate the VIS (Figure 3). It is the weight that CMS places on each domain (HCAHPS, clinical processes of care, outcomes, etc.) and is shown in Table 2. A higher weight by CMS results in a larger multiplier, indicating a larger impact on the TPS. The VBP weight is defined for each measure, using the equation: 


\begin{tabular}{|c|c|}
\hline VA Model HFMEA & VA Model HFMEA \\
Mode 1 Failure \\
Fode 2
\end{tabular}

Figure 1: Applying the HFMEA model to value based purchasing. Note: HFMEA: Health Failure Modes Effects Analysis; VA: Veterans Affairs; VBP: Value Based Purchasing; VFMEA: Value Failure Modes Effects Analyses.

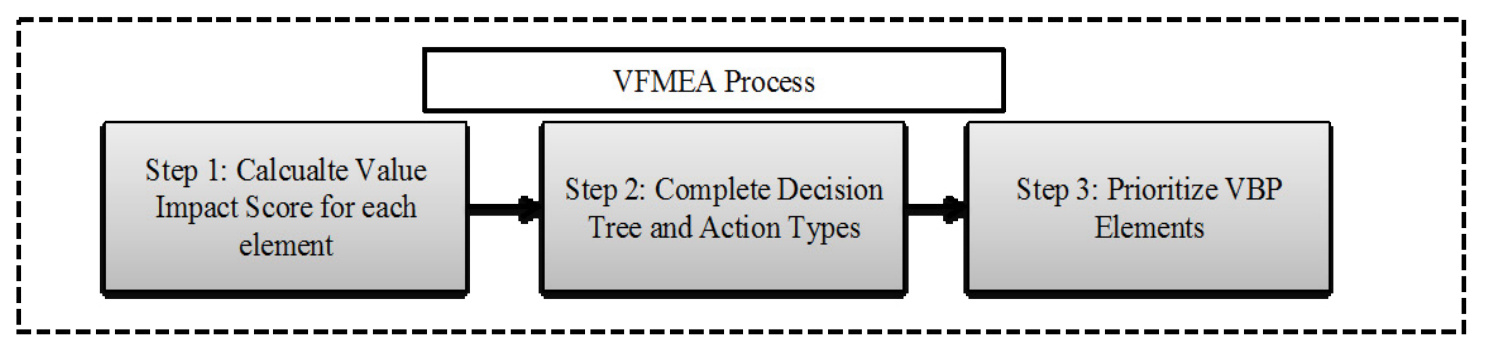

Figure 2: VFMEA workflow method. Note: From US Department of Veterans Affairs (va), National center for patient safety healthcare failure modes and effects analysis [11].

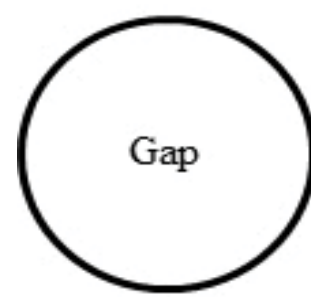

VBP Weight $=\frac{\text { Domain Weight }}{\text { Number of Elements in Domain }}$

\section{Step 2: Complete the VFMEA decision tree and action types}

To make the VA's HFMEA applicable to VBP, only minimal modifications to the wording in the decision tree were needed. A comparison of the VA questions and the new VFMEA decision tree questions is shown in Table 3, and the decision tree logic is presented in Figure 4. The decision tree did not need to be piloted, as it was already in use in healthcare and has been used in hospitals across the VA system [7].

HFMEA includes three action types for identified failure modes: accept, eliminate, or control [7]. In the VFMEA process, accepting the failure mode remains a valid action type, as hospital leaders can chose not to take any action to improve performance on a particular VBP measure. Eliminating a potential failure mode is not a valid option in the VFMEA structure, as the measures are required by CMS, and hospital leadership does not have the discretion to remove a measure from required VBP reporting. For this reason, the VFMEA process did not include eliminate as a potential action type. Controlling the failure mode remained a valid action type. Hospital leaders can chose to make process changes that will improve the hospital's performance on a particular VBP measure. In making the decision to control a VBP measure, hospital leaders should consider factors unique to the hospital such as risk of improving/not improving the measure, how aligned the measure is with other strategic objectives, the complexity of the improvement effort, and any other facility-specific characteristics or environmental factors.

In a typical HFMEA, outcome measures would be identified for each failure mode in which the selected action type was either eliminate or control [7]. With VBP, CMS clearly defined the outcome for each measure so hospital-specific outcome measures were not necessary in the VFMEA. Hospital leaders can measure outcomes as improvements to individual measures over time or improvements to the TPS over time.

\section{Step 3: Prioritize value based purchasing elements}

Using VFMEA templates, this case study prioritized VBP measures. VBP measures with control as the action type were listed in descending 
Citation: Cress A, Revere L, Mikhail O, Pompeii L, Simmons D (2017) Engineering Value in Healthcare: Applying Failure Modes and Effects Analysis to Value Based Purchasing. Health Econ Outcome Res Open Access 3: 124. doi: 10.4172/2471-268x/1000124

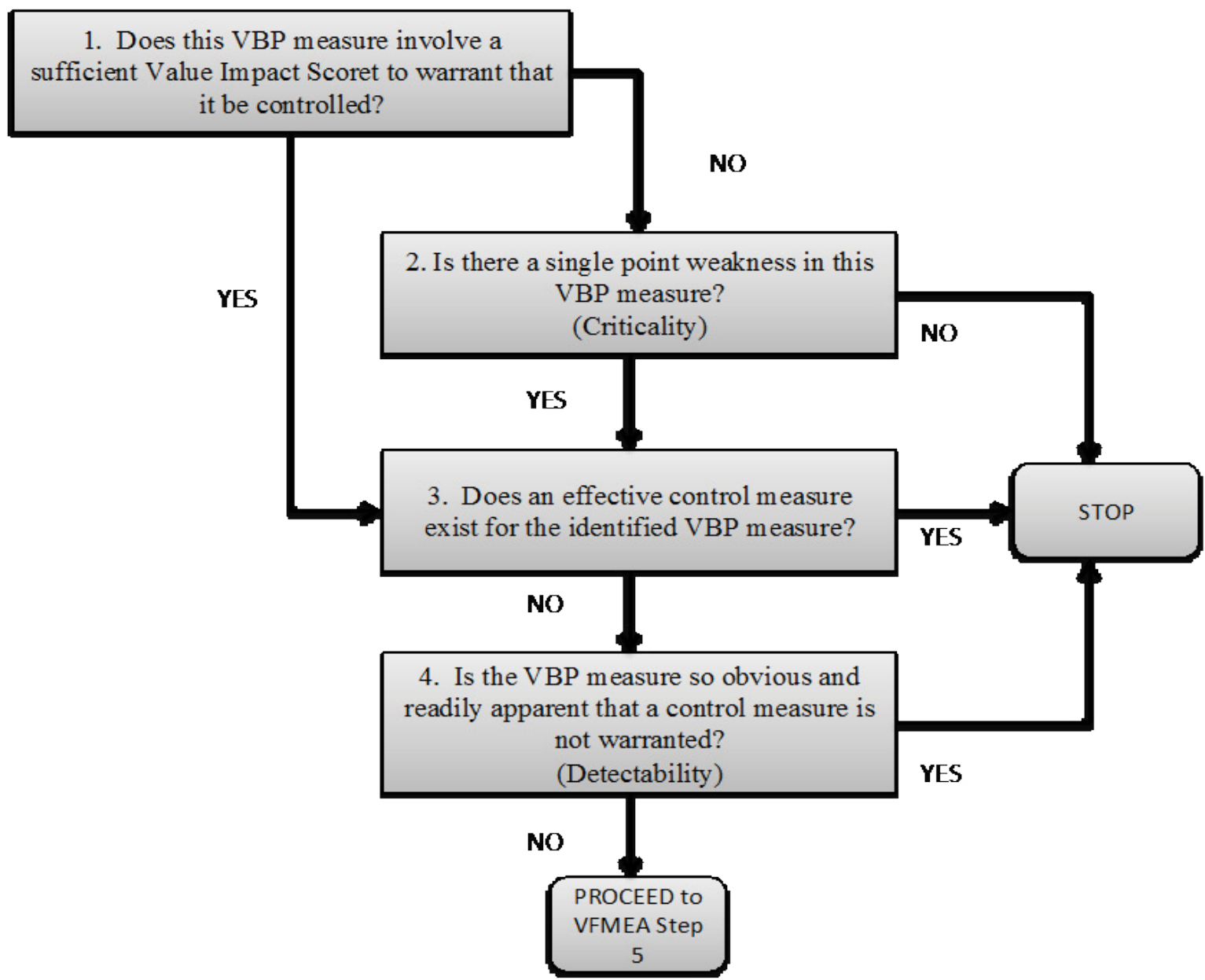

Figure 4: VFMEA Decision Tree. Note. VA: Veterans Affairs; VBP: Value Based Purchasing; VIS: Value Impact Score; VFMEA: Value Failure Modes and Effects Analysis.

\begin{tabular}{|c|l|}
\hline Question Number & VA HFMEA Decision Tree Questions \\
\hline 1 & $\begin{array}{l}\text { Does this hazard involve a sufficient likelihood and severity to } \\
\text { warrant that it be controlled? (e.g. hazard score higher than } 8 \text { ) }\end{array}$ \\
\hline 2 & $\begin{array}{l}\text { Is there a single point weakness in the process? (e.g. failure will } \\
\text { result in a system failure) } \\
\text { (Criticality) }\end{array}$ \\
\hline 3 & $\begin{array}{l}\text { Does an effective control measure exist for the identified hazard? } \\
\text { Is the hazard so obvious and readily apparent that a control } \\
\text { measure is not warranted? } \\
\text { (Detectability) }\end{array}$ \\
\hline 4
\end{tabular}

\section{VFMEA Decision Tree Questions}

Does this VBP measure involve a sufficient gap and weight to warrant that it be controlled? (e.g. VIS greater than 50 )

Is there a single point weakness in this VBP measure? (e.g. failure of the measure will results in failure in the overall VBP Program) (Criticality)

Does an effective control measure exist for the identified VBP measure? Is the VBP measure so obvious and readily apparent that a control measure is not warranted?

(Detectability)

Note: HFMEA: Healthcare Failure Modes and Effects Analysis; VA: Veterans Affairs; VBP: Value Based Purchasing; VIS: Value Impact Score; VFMEA: Value Failure Modes and Effects Analysis. Adapted from U.S. Department of Veterans Affairs (VA). National Center for Patient Safety [11].

\section{Table 3: HFMEA and VFMEA decision tree comparison.}

order by their VIS. The result was two separate lists. The first was a list of measures with a low VIS, indicating the hospital should maintain their processes and reevaluate in future VFMEA cycles. Recall, the VIS is a multiplier reflecting the weight assigned to the measure in VBP and the hospital's potential room for improvement on the measure. The second was a list of measures with high VIS and control action types, indicating the projects with the ability to have the largest impact on overall score, and thus areas in which the hospital should direct performance improvement projects. This list was the input for the resource allocation worksheets.

\section{Results}

This research reports the results of a case study application of the VFMEA at a pilot hospital. The hospital is a 154 bed, non-profit, general community hospital in the suburbs of Houston, Texas. The average daily census is approximately 100 ; there are 9,881 annual adult admissions and approximately 38,000 emergency room visits. The pilot hospital is representative of typical community hospitals, thus this research should be generally applicable to community hospitals seeking to increase their TPS scores. 
The case study was conducted during two, 3 hour meetings. The case study team was the same for both meetings; it consisted of the chief nursing officer, director of medical/surgical nursing, director of critical care, director of women's services, and director of quality. The team actively participated in the process throughout the two meetings by responding to decision tree questions, engaging their peers in meaningful debate, and challenging each other's answers or ideas in order to reach consensus. All data to calculate the VIS was obtained from the CMS Medicare Compare website (https://data.medicare.gov/).

The VIS was calculated for each measure according to the method outlined in Step 1. VIS ranged from 83.3 to 0. VIS for outcomes and HCAHPS results were consistently higher than VIS for clinical processes of care, suggesting improvements in outcomes and/or HCAPHS will have a larger impact than improvements in clinical processes of care. Thirty-day mortality rates for acute myocardial infarction, heart failure, and pneumonia tied for the highest VIS at 83.3. Five of the eight HCAHPS measures scored 37.5, with two HCAHPS measures scoring 33.8. All measures for clinical processes of care scored below 31.8 with seven scoring below 10.4 .

The case study team used the decision tree shown in Figure 4 to further assess each measure. The decision tree provided a structured series of questions for the team to determine if the VIS warranted further action and whether that action should be to stop, accept, or control the measure. Each member of the case study team was provided with a copy of the decision tree as outlined in Step 3 of the methodology. The decision tree was reviewed for all 24 measures.

Using the decision tree, the case study team identified 15 measures that required a decision on the action type - either accept or control. Each member of the case study team was provided definitions for accept and control as outlined in Step 3 of the methodology. The team decided to accept 3 measures and control 12. For the three measures the team accepted, no further action was taken. The team completed a resource allocation worksheet for each of the 12 measures they decided to control. These 12 measures are outlined in Table 4.

\section{Conclusion}

The complexity of VBP poses a management challenge to healthcare leaders. It is difficult for leaders to prioritize VBP measures because of multiple domains, multiple measures, and changes implemented every fiscal year, changing break-even points every fiscal year, and the challenges of calculating the TPS. VanLare et al. confirms the current need of healthcare providers both to identify opportunities and make quality improvements to VBP measures [9]. Currently, there is no objective method for hospital leaders to prioritize the VBP measures and identify those opportunities that can have the largest impact on their TPS. Looking outside of the healthcare industry, this research has developed and piloted a proven methodology for prioritizing improvement opportunities based on their financial impact.

Through the VBP program, CMS has provided a definition and structure for measuring value in hospital-based healthcare services. The VFMEA developed in this research is a structured, objective method for prioritizing the measures of VBP that can be easily applied by any hospital. Using VFMEA, leaders can more effectively allocate resources to improving those VBP elements that will maximize the reimbursement the hospital receives from VBP performance. It provides hospital leaders an objective method for comparing opportunities for improvement that otherwise would be challenging, such as comparing clinical process of care or outcomes to opportunities for improvement in patient satisfaction. Improvements in the VBP score means patients

\begin{tabular}{|c|}
\hline Domains and Measures \\
\hline Clinical Processes of Care \\
Discharge Instructions \\
\hline Prophylactic Antibiotic Received Within One Hour Prior to Surgical Incision \\
\hline HCAHPS \\
\hline Discharge Information \\
\hline Hospital Cleanliness and Quietness \\
\hline Hospital Staff Responsiveness \\
\hline Medicine Communication \\
\hline Nurse Communication \\
\hline Overall Hospital Rating \\
\hline Pain Management \\
\hline Outcomes \\
\hline Acute Myocardial Infarction 30-Day Mortality Rate \\
\hline Heart Failure 30-Day Mortality Rate \\
\hline Pneumonia 30-Day Mortality Rate \\
\hline
\end{tabular}

Table 4: Measures selected by case study team to be controlled.

are experiencing better results, be it HCHAPS, clinical process of care, or outcomes, as the net effect to patients is positive. Hospital leaders making the decision to control, rather than improve, a VBP measure, should consider factors unique to the hospital, such as risk of improving/not improving the measure, how aligned the measure is with other strategic objectives, the complexity of the improvement effort, and any other facility specific characteristics or environmental factors.

Thus, VFMEA represents a unique approach to assessing opportunities for improvement in VBP by applying a proven engineering methodology to a specific challenge in healthcare quality. Hospital leaders are provided structured guidance on to make changes that will improve the hospital's performance on a particular VBP measure and the result economic gain. As a result of this research, hospital leaders now have a low cost, effective tool for identifying where to direct resources to achieve the largest gain in VBP incentive payments.

\section{References}

1. Hospital Value Based Purchasing (VBP) program (2013) 42 C.F.R. 410.

2. VanLare JM, Conway PH (2012) Value Based Purchasing national programs to move from volume to value. N Engl J Med 367: 292-295.

3. US Office of Legislative Counsel (2010) Compilation of Patient Protection and Affordable Care Act, as amended through May 1, 2010, including Patient Protection and Affordable Care Act Health-Related Portions of the Heath Care and Education Reconciliation Act of 2010. Rpt 111-1.

4. Hospital Value Based Purchasing

5. Stalhandske E, DeRosier J, Patail B, Gosbee J (2003) How to make the most of failure mode and effect analysis. Biomedical Instrumentation and Technology/ Association for the Advancement of Medical Instrumentation 37: 96-102.

6. Stalhandske E, DeRosier J, Wilson R, Murphy J (2009) Healthcare FMEA in the Veterans Health Administration. Patient Saf Qual Health 20: 33.

7. DeRosier J, Stalhandske E, Bagian JP, Nudell T (2002) Using health care failure mode and effect analysis ${ }^{\mathrm{TM}}$ : The VA National Center for Patient Safety's Prospective Risk Analysis System. Jt Comm J Qual Patient Saf 28: 248-267.

8. DeRosier J, Nudell T (2001) The Basics of Healthcare Failure Mode and Effect Analysis. VHA Video teleconference broadcast from Ann Arbor, Michigan.

9. VanLare JM, Williams JM, Conway PH (2012) Value Based Purchasing for hospitals. Health Affairs (Project Hope) 31: 249.

10. The Medicare Learning Network ${ }^{\circledR}$ (MLN) (2013b) "Fact sheet: Hospital Value Based Purchasing Program ICN 907664.

11. US Department of Veterans Affairs (VA) (2013) National Center for Patient Safety. Healthcare failure modes and effects analysis (HFMEA). 